\title{
Global stroke statistics: An update of mortality data from countries using a broad code of "cerebrovascular diseases"
}

\author{
Amanda G Thrift ${ }^{1}$, George Howard ${ }^{2}$, Dominique A Cadilhac ${ }^{1,3}$, Virginia J Howard ${ }^{4}$, Peter M \\ Rothwell ${ }^{5}$, Tharshanah Thayabaranathan ${ }^{1}$, Valery L Feigin ${ }^{6}$, Bo Norrving ${ }^{7}$ and Geoffrey A Donnan ${ }^{2,8}$
}

\begin{abstract}
Background: Current information on mortality attributed to stroke among different countries is important for policy development and monitoring prevention strategies. Unfortunately, mortality data rep orted to the World Health Organization by different countries are inconsistent.
\end{abstract}

Aims and/or hypothesis: To update the repository of the most recent country-specific data on mortality from stroke for countries that provide data using a broad code for "cerebrovascular dis ease."

Methods: Data on mortality from stroke were obtained from the World Health Organization mortality database. We searched for countries that provided data, since 1999, on a combined category of "cerebrovascular disease"(code 1609) that incorporated International Classification of Diseases (10th edition) codes I60-I69. Using population denominators provided by the World Health Organization for the same year when available, or alternatively estimates obtained from the United Nations, we calculated crude mortality from "cerebrovascular disease" and mortality adjusted to the World Health Organization world population. We used the most recent year reported to the World Health Organization, as well as comparing changes over time.

Results: Since 1999, seven countries have provided these mortality data. Among these countries, crude mortality was greatest in the Russian Federation (in 2011), Ukraine (2012), and Belarus (2011) and was greater in women than men in these countries. Crude mortality was positively correlated with the proportion of the population aged 65 years but not with time. Age-adjusted mortality was greatest in the Russian Federation and Turkmenistan, and greater in men than women. Overtime, mortality declined, with the greatest decline per annum evident in Kazakhstan (8.7\%) and the Russian Federation (7.0\%).

Conclusions: Among countries that provided data to the World Health Organization using a broad category of "cerebrovascular disease," there was a decline in mortality in two of the countries that previously had some of the largest mortality rates for stroke.

Keywords: Mortality, global, worldwide, stroke, burden, epidemiology

\section{Introduction}

With the growing burden of stroke globally, an understanding of its characteristics and impact in as many countries as possible is important for monitoring the success of prevention strategies and to develop policies to reduce its impact. In our 2017 update on global stroke statistics published in the International Journal of Stroke (IJS), ${ }^{1}$ we included countries that provided mortality data to the World Health Organization (WHO) on individual categories of stroke according to the International Classification of Diseases (ICD; 10th edition) codes I60, I61, I62, I63, I64, I67, and I69, as well as individual codes for ICD-8 and ICD-9. Some other countries provide their mortality data using a combined broad category of stroke that include these codes but also include codes I65, I66, and I68. These additional codes are for occlusion and stenos is of pre-cerebral (I65) and cerebral (I66) arteries not resulting in cerebral infarction, and cerebrovascular disorders in diseases classified elsewhere (I68). We determined that including data from these countries may not have provided a realistic comparison, as their mortality statistics for stroke would likely be overestimated (i.e. since non-stroke codes are potentially included). Nonetheless, these data are relevant to report separately where no other 
information is available. This report provides mortality data on countries that used this broad category of "cerebrovascular disease".

\section{Aim}

To update the IJS repository of the most recent country-specific data on mortality from stroke for countries that provide data using a broad code for "cerebrovascular disease".

\section{Methods}

Data on mortality from cerebrovascular disease were obtained from the WHO mortality database at www.who.int/healthinfo/statistics/mortality_rawdata/ en/. These deaths comprise those that have been coded by the national authority within each country and are then provided to the WHO. We searched for countries that provided data, since 1999, on a combined category of "cerebrovascular dis ease" (code 1609) that incorporated ICD-10 codes I60-I69. We used the data that were updated on 25 November 2015, as these data provide a contemporaneous comparison with those used in our last review. ${ }^{1}$ The files used comprise population denominators, country codes, and mortality data from the ICD-10 classification. When population denominators were not available, we used the estimates of the population obtained from the United Nations for the closest available year (https://esa.un.org/unpd/wpp/ Download/Standard/Population/).

We merged data on deaths from cerebrovascular dis ease with the population denominators for the same year as that in which mortality was coded. Crude annual mortality of stroke was obtained by dividing the overall deaths from cerebrovascular diseases for each country per year by the total population for that year. These were then multiplied by 100,000 to obtain crude mortality per 100,000 population per year. The same calculations were conducted for men and women, separately. We then calculated age-adjusted death rates, overall and for each sex separately, standardized to the WHO world population using the direct method. ${ }^{2}$ We used the largest number of categories for our age-standardization calculations, i.e. five-year age bands when available, but otherwise we used 10-year age bands. The upperage bands ranged from 75 plus years to 95 plus years. We report the most recent data for each country.

We undertook a regression analysis of crude mortality versus the proportion of the population aged ?65 years, as previously. ${ }^{3}$ We repeated this analysis for crude mortality by year. To determine whether there had been changes in age-adjusted mortality over time we also undertook linear regression analysis of age-adjusted mortality for each country that had mortality data for more than five years.
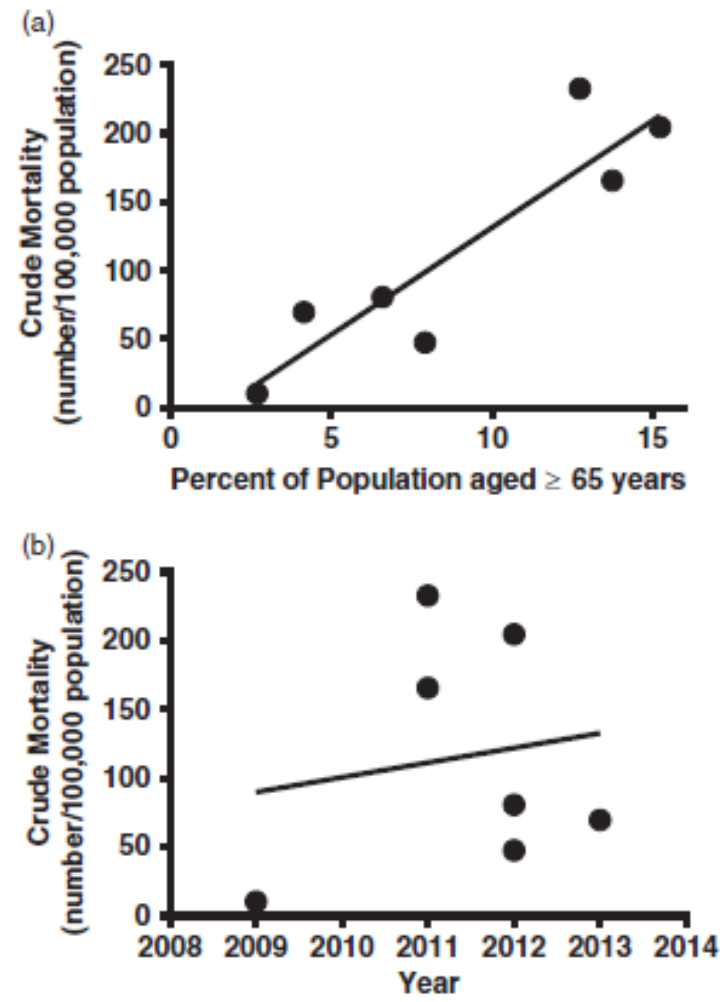

Figure 1. Crude mortality from stroke according to (a) the proportion of the population aged at least 65 years (Y1/415.61X-24.69, $\mathrm{p}^{1 / 40.0048)}$ and (b) the year

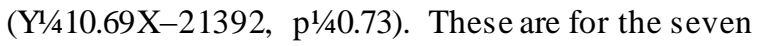
countries that have reported mortality to the World Health Organization using a broad category of "cerebrovascular disease". 


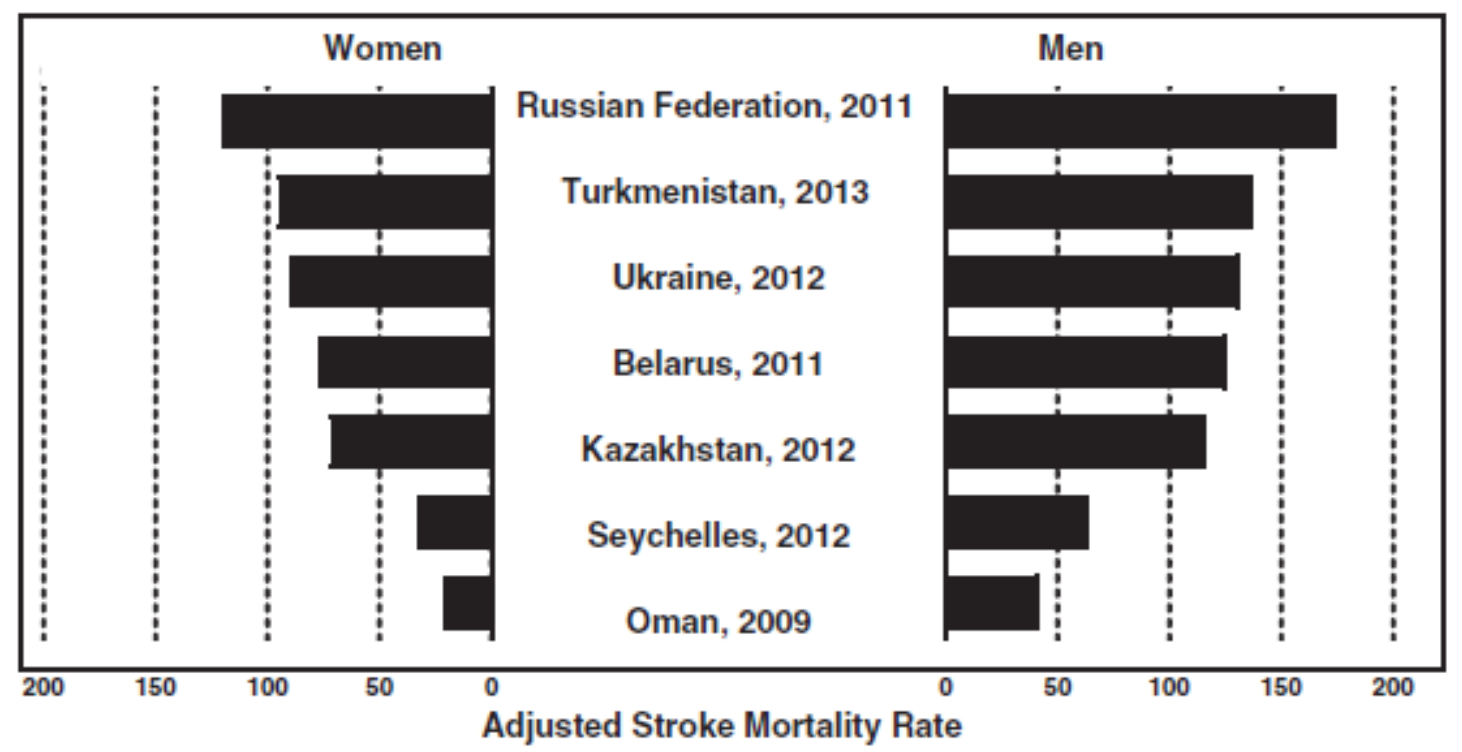

Figure 2. Age-adjusted mortality from stroke in the most recent year reported to the World Health Organization, ordered according to the average mortality for men and women.

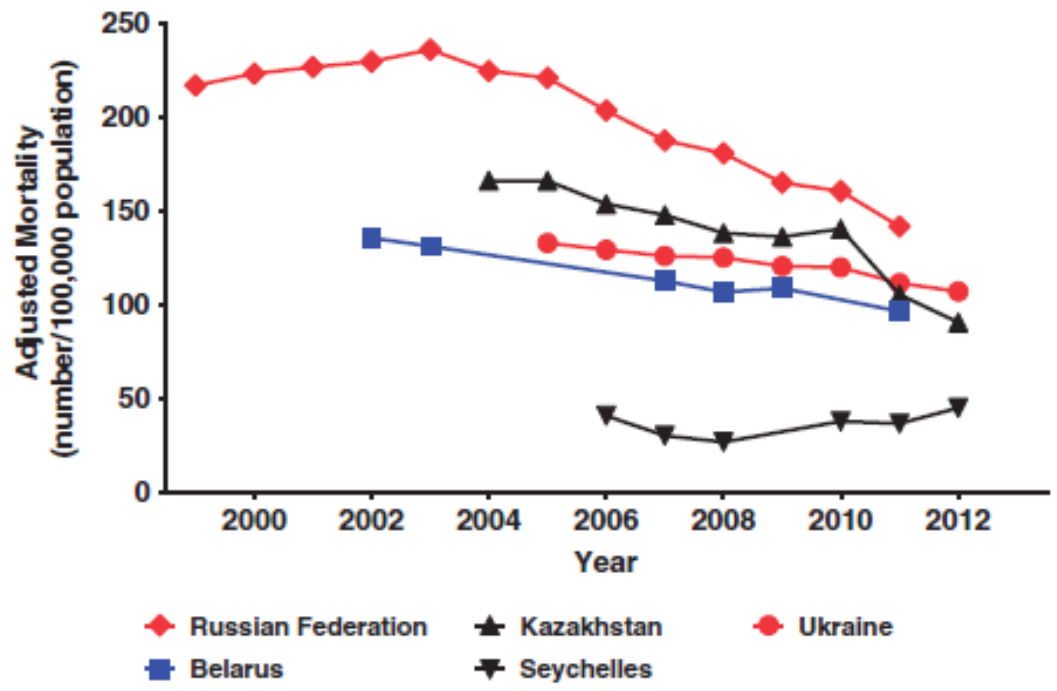

Figure 3. Mortality age adjusted to the World Health Organization population. ${ }^{2}$ 

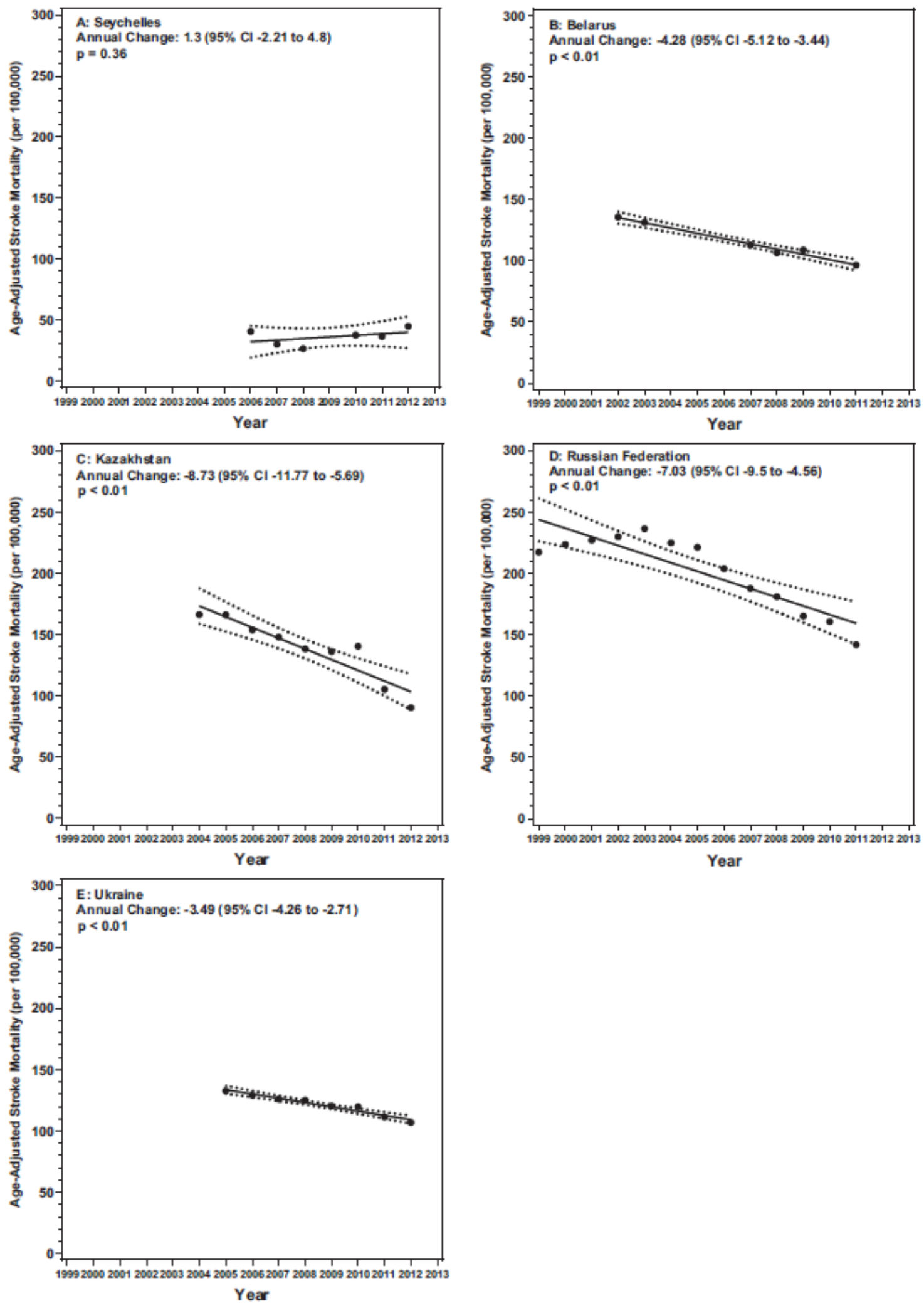

Year

Figure 4. Annual percentage change in age-adjusted mortality of stroke for Seychelles (A), Belarus (B), Kazakhstan (C), Russian Federation (D), and Ukraine (D). Data obtained from the World Health Organization mortality database (code 1609; cerebrovascular disease). 


\section{Results}

We identified seven countries that had provided data to the WHO that incorporated a broad category of "cerebrovascular disease" for at least one year since 1999: Belarus, Kazakhstan, Oman, Russian Federation, Seychelles, Turkmenistan, and Ukraine. All of these countries, except Oman, had previously provided data to the WHO in stroke-specific codes.

Among these seven countries, crude mortality was greatest in the Russian Federation (in 2011), Ukraine, and Belarus, with crude mortality greater in women than men in all three countries.

There was a positive correlation between crude mortality and the proportion of the population aged 65 years ( $\mathrm{Y}$ $=15.61 \mathrm{X}-24.69, \mathrm{p}=0.0048)$ but not with year $(\mathrm{Y}=10.69 \mathrm{X}-21392, \mathrm{p}=0.73$; Figure 1). Given the proportion of the population aged 65 years (12.7\%), the Russian Federation had a greater mortality than expected (233/100,000/year). In contrast, the Seychelles had a crude mortality from stroke (48/ 100,000/year) less than expected given the proportion of people aged 65 years $(7.9 \%)$.

Age-adjusted mortality was greatest in the Russian Federation and Turkmenistan, being greater in men than in women (Figure 2). Over time, mortality declined for Belarus, Kazakhstan, the Russian Federation, and Ukraine (Figure 3). The greatest decline was evident in Kazakhstan (annual change $-8.73 \%, 95 \%$ CI: -11.77 to -5.69 ) and in the Russian Federation (annual change -7.03\%, 95\% CI: -9.5 to -4.56; Figure 4).

\section{Discussion}

We identified seven countries that provided mortality data on stroke to the WHO that included a broad cat egory of "cerebrovascular disease." Six of these countries had previously used stroke-specific codes. Because the current mortality data included codes I65, I66, and I68 together with stroke, mortality rates for this category are potentially greater than for stroke-specific codes alone. Therefore, it was important to report mortality for these countries separately, so that mortality rates were not unfairly compared between countries. Nonetheless, variation in the codes used limits the comparisons that can be made among different countries. In our original paper, 123 countries used consistent mortality coding and provided a much richer source of information for reporting differences in stroke mortality rates around the world. ${ }^{3}$

In this additional report, the countries that had the greatest crude mortality from stroke had more strokes in women than in men. The fact that the age-adjusted mortality rates were greater in men than women in these countries simply demonstrates that women have their strokes at older ages than men. Indeed, these seven countries had a much greater proportion of their population aged at least 65 years, a measure that is highly correlated with crude mortality.

Our finding that mortality rates have declined for the two countries with the greatest mortality from stroke, the Russian Federation and Kazakhstan, is encouraging. This decline suggests that these countries are undertaking health initiatives targeted at either prevention, treatment or both, or that other factors unrelated to healthcare are involved. As there have been no new incidence studies of stroke in these countries for more than a decade, ${ }^{4,5}$ it is unknown whether incidence is also declining. Therefore, it remains unclear whether this decline in mortality is mirroring a decline in incidence, or whether the main driver is a reduction in case fatality.

There are some limitations to this study. Official mortality statistics are unreliable and may be influenced by factors such as between-country differences in death coding practice and changes in death coding practice over time. Indeed, because of these variations in coding practices it is unclear how much of an influence the extra codes may have on mortality figures, if any. It is possible that the code for "cerebrovascular dis -ease"may include elderly people who may have had cognitive problems, memory impairment, or were diag nosed with socalled atherosclerosis of the brain arteries during their lives. However, the actual practices in coding these data are unclear. The Global Burden of Diseases Study has overcome some of these is sues in coding by estimating mortality using the Cause of Death Ens emble model (CODEm). ${ }^{6}$ In this highly computational model different combinations of regression models are evaluated against death rates; fractions of death attributable to a specific 
cause; and data on risk factors such as tobacco use, metabolic, socioeconomic, and various dietary risks. ${ }^{7}$ However, estimates that are derived using CODEm require substantialcomputing power, ${ }^{6}$ which is not available in many areas of the world.

Data linkage between hospital and mortality data could provide a useful way for countries to determine whether incidence of stroke is declining. ${ }^{8}$ This procedure can also be used to evaluate quality improvement programs ${ }^{9}$ and plan services ${ }^{10}$ However, these types of data are best used in regions where the majority of people with stroke are hospitalized. In regions where many people with stroke are not hospitalized, other means for capturing episodes of stroke outside the hos pital system are required. The significant problem of incorrect coding of incident stroke cases, even in hos pitalized patients, ${ }^{11}$ must also be taken into account when interpreting apparent temporal time trends in stroke incidence and mortality.

\section{Conclusion}

Mortality data for stroke from some countries should be reported separately to ensure that comparisons between countries are not unfair. Ideally, these countries should be encouraged to provide the same codes so that more consistent reporting can occur. The finding of a decline in stroke mortality in two of the countries that previously had some of the most elevated mortality from stroke is noteworthy. Linkage between hospitaldata and death records is likely to provide a useful way to help identify whether the decline in mortality from stroke is attributable to better treatment or reduction in incidence, and may complement more rigorous data from repeat incidence studies.

\section{Acknowledgments}

The source of the mortality data is the World Health Organisation (WHO) Mortality database, and the WHO is responsible only for the provision of the original information. The analyses, interpretations, and conclusions are the authors alone, and not the WHO.

\section{Declaration of conflicting inte rests}

The author(s) declared no potential conflicts of interest with respect to the research, authorship, and/or publication of this article.

\section{Funding}

The author(s) disclosed receipt of the following financial support for the research, authorship, and/or publication of this article: The following authors receive Research Fellowship support from the National Health and Medical Research Council: Dominique Cadilhac (1063761; cofunded by Heart Foundation) and Amanda Thrift (1042600). Tharshanah Thayabaranathan received an Australian Postgraduate Award.

\section{References}

1. Thrift AG, Thayabaranathan T, Howard G, et al. Global stroke statistics. Int J Stroke 2017; 12: 13-32.

2. Ahmad OB, Boschi-Pinto C, Lopez AD, Murray CJL, Lozano R and Inoue M. Age standardization of rates: a new WHO world standard. Geneva: World Health Organization, 2001 (GPE discussion paper series: No.31, http://www.who.int/healthinfo/paper31.pdf?ua $\underline{1} \underline{4} \underline{1}$, accessed 21 June 2017).

3. Thrift AG, Cadilhac DA, Thayabaranathan T, et al. Global stroke statistics. Int J Stroke 2014; 9: 6-18.

4. Feigin VL, Wiebers DO, Nikitin YP, O'Fallon MW and Whisnant JP. Stroke epidemiology in Novosibirsk, Russia: a population-based study.Mayo Clinic Proc 1995; 70: 847-852.

5. Mihalka L, Smolanka V, Bulecza B, Mulesa S and Bereczki D. A population study of stroke in West Ukraine: incidence, stroke services, and 30-day case fatality. Stroke 2001; 32: 2227-2231.

6. Roth GA, Johnson CO, Nguyen G, et al. Methods for estimating the global burden of cerebrovascular diseases. Neuroepidemiology 2015; 45: 146-151.

7. Foreman KJ, Lozano R, Lopez AD and Murray CJ. Modeling causes of death: an integrated approach using CODEm. Popul Health Metr 2012; 10: 1. 
8. Nedkoff L, Briffa TG, Knuiman M, et al. Temporal trends in the incidence and recurrence of hospitalised atherothrombotic disease in an Australian population, 2000-07: data linkage study. Heart 2012; 98: 1449-1456.

9. Ido MS, Bayakly R, Frankel M, Lyn R and Okosun IS. Administrative data linkage to evaluate a quality improvement program in acute stroke care, Georgia, 2006-2009. Prev Chronic Dis 2015; 12: E05.

10. Katzenellenbogen JM, Vos T, Somerford P, Begg S, Semmens JB and Codde JP. Burden of stroke in indigenous Western Australians: a study using data linkage. Stroke 2011; 42: 1515-1521.

11. Li L and Rothwell PM. Biases in detection of apparent "weekend effect" on outcome with administrative coding data: population based study of stroke. BMJ 2016; 353: i2648. 